\begin{tabular}{|c|l|}
\hline Title & Modification of statocyst input to local interneurons by behavioral condition in the crayfish brain \\
\hline Author(s) & Hama, N.; Takahata, M. \\
\hline Citation & $\begin{array}{l}\text { Journal of Comparative Phy siology A: Neuroethology, Sensory, Neural, and Behavioral Physiology, 191(8), 747-759 } \\
\text { https://doi.org/L0.1007/300359-005-0630-z }\end{array}$ \\
\hline Issue Date & $2005-08$ \\
\hline Doc URL & http://hdl.handle.net/2115/16954 \\
\hline Rights & The original publication is available at www.springerlink.com \\
\hline Type & article (author version) \\
\hline File Information & JCPA-NSN\&BP191-8.pdf \\
\hline
\end{tabular}

Instructions for use 


\title{
Modification of Statocyst Input to Local Interneurons by Behavioral Condition in the Crayfish Brain
}

\author{
N. Hama* and M. Takahata \\ Animal Behavior and Intelligence, Division of Biological Sciences, Graduate School of \\ Science, Hokkaido University, Sapporo 060-0810, Japan
}

*Corresponding author

Tel: $\quad+81-11-706-2753$

Fax: $\quad+81-11-706-4923$

E-mail: hnori@sci.hokudai.ac.jp 


\section{Abstract}

Posture control by statocysts is critically affected by leg condition in decapod crustaceans. We investigated in the crayfish brain how the synaptic response of local interneurons to statocyst stimulation was affected by leg movements on and off a substratum. In order to stimulate statocyst receptors as naturally as possible, we adopted the magnetic field stimulation method that enabled sustained stimulation of statocyst receptors by mimicking body rolling. The statocyst-driven local interneurons were classified into 4 morphological groups (Type-I - IV). All interneurons except Type-IV projected their dendritic branches to the parolfactory lobe of the deutocerebrum where statocyst afferents project directly. Type-I interneurons having somata in the ventral paired lateral cluster responded invariably to statocyst stimulation regardless of the leg condition whereas others having somata in the ventral unpaired posterior cluster showed response enhancement or suppression, depending on the cell, during leg movements on a substratum, but no response change during free leg movements off the substratum. The synaptic responses of Type-II and IV interneurons were also affected differently by leg movements depending on the substratum condition whereas those of Type-III remained unaffected. These findings suggest that the statocyst pathway in the crayfish brain is organized in parallel of local circuits that are affected by leg condition and those not affected. 
Key Words: Statocyst, Local interneurons, Crayfish, Brain, Response modulation, Multimodal integration

\section{Abbreviations}

NGI: Nonspiking Giant Interneurons (Okada and Yamaguchi 1988)

SDI: Statocyst Descending Interneurons (Hama and Takahata 2003)

EUMN, EDMN: Eyecup Up (Down) Motor Neuron (Furudate et al. 1996) 


\section{Introduction}

Interaction among equilibrium, visual and somatosensory systems crucially affects the control of animal body posture and orientation in vertebrates (Ullén et al 1995; Horak and Macpherson 1996; Deliagina et al. 1999) and invertebrates (Schöne et al 1983; Okada et al 1994). In addition, ongoing activity and behavioral context of the animal also affect this interaction significantly (Davis et al 1974; Everett et al 1982; Deliagina et al 2000). However, the synaptic mechanisms subserving the intermodal sensory and central interactions largely remain unknown.

In crustaceans, body tilting activates statocyst receptors depending on the tilt direction and magnitude to evoke equilibrium responses involving eyestalks, walking legs and uropods (Kühn 1914; Schöne 1954; Davis 1968; Yoshino et al 1980). Leg proprioceptive and visual inputs critically affect these responses (Kühn 1914; Alverdes 1926; Schöne et al 1976). The interaction among these sensory inputs is highly complex that the direction of uropod steering response is completely reversed depending on whether the legs are provided with a substratum or not (Takahata et al 1984). Another line of evidence for the importance of sensory interaction in postural control comes from the study of "central compensation" (Schöne, 1954). Unilateral statolith removal results in asymmetrical posture of eyestalks and uropods (Schöne 1954; Yoshino et al 1980). The symmetrical posture is recovered 2 weeks after removal when a substrate is provided, but hardly recovered if it is not provided (Sakuraba and Takahata 1999, 2000). 
In addition, it has been known that the statocyst information controlled by proprioceptors is able to induce a swimming in the crab (Fraser et al. 1987). These reports suggest that the statocyst pathway makes critical interaction with sensory inputs from leg proprioceptors or central signals related to leg movements.

Neuronal elements involved in the statocyst pathway within the brain have been studied extensively. The crayfish brain consists of three major parts: protocerebrum, deutocerebrum and tritocerebrum (Sandeman et al. 1992). The protocerebrum is the region where nonspiking giant interneurons (NGIs) that function as the premotor element in compensatory eyestalk movements are located (Yamaguchi and Okada 1990) and where statocyst descending interneurons (SDIs) project extensive dendrites (Hama and Takahata 2003). The deutocerebrum includes the parolfactory lobe where the statocyst afferents project directly (Yoshino et al. 1983). The tritocerebrum includes antennal and tegumentary lobes with less projections of SDIs than in the protocerebrum: some SDIs project dendrites only to the protocerebrum (Hama and Takahata, 2003). Synaptic responses of SDIs that are organized in parallel are differently affected by ongoing activity and leg condition to enable behavioral context dependent posture control, confirming the results of preceding studies (Takahata and Hisada 1982; Nakagawa and Hisada 1989; Fraser and Takahata 2002). In the protocerebrum, NGIs respond to multimodal sensory inputs including statocyst, leg proprioceptor and visual signals (Okada et al. 1994; Furudate et al. 1996). Although 
Nakagawa and Hisada (1990) reported local interneurons having dendrites in the parolfactory lobe and receiving presumably monosynaptic input from the statocyst afferents, it remains largely unknown how the statocyst information is transmitted from the parolfactory lobe in the deutocerebrum to the protocerebrum where NGIs and dendrites of SDIs are located, and how it interacts with other sensory and central information.

In the present study, we addressed a question how the local pathway connecting the deuto- and protocerebrum is organized, and made intracellular recording from statocyst-driven local interneurons to analyze the effect of leg proprioceptive input and behavioral condition on their responses. Based on their morphological characteristics revealed by intracellular dye injection, we propose a hypothetical wiring diagram connecting the deuto- and protocerebrum for synaptic transmission of statocyst information. 


\section{Materials and methods}

Experimental animals and procedures, relating to intracellular recording and staining, extracellular recording from the circumesophageal commissure, and leg movement monitoring by force transducers, were the same as those reported in our previous study (Hama and Takahata 2003). In short, adult crayfish Procambarus clarkii of both sexes were used. Four weeks before experiment, statoliths were replaced with ferrite grains on both sides. The rostrum was cut away and the nonsensory hairs covering the opening of the statocyst were removed with fine forceps. Then the statolith was washed out with water jet using a fine glass pipette. Ferrite grains were introduced into the statocyst lumen using a fine needle. After this operation, animals were kept in laboratory tanks before experiment for four weeks. Before dissection, the chelipeds were cut away and each crayfish was anesthetized in cooled physiological saline (van Harreveld 1936). A small portion of dorsal carapace was removed to reveal the brain. Then the animal was fixed dorsal side-up in the air to a metal rod framework. All legs and the abdomen were kept free to move.

In order to monitor the leg activity, electromyographic (EMG) recording was made from the mero-carpopodite flexor muscle of the second or third walking leg on the side of stimulation. Since our preliminary EMG analyses of leg movements during walking revealed that, in order to draw any definite conclusion on leg movements, EMG recording has to be made from all 8 legs simultaneously, we reserved in this study any 
further consideration of EMG records beyond whether the leg was actively moving or not.

Details on the magnetic field stimulation are described elsewhere (Ozeki et al. 1978; Takahata and Murayama 1992). In some experiments, we used Alexa Fluor 488 (Molecular Probes AF488, $10 \mathrm{mM}$ in $200 \mathrm{mM} \mathrm{KCl}$ ) for intracellular staining instead of Lucifer Yellow. AF488 was injected into neurons by applying negative current pulses (up to $7 \mathrm{nA}, 500 \mathrm{~ms}$ duration, $1 \mathrm{~Hz}$ for $10-15 \mathrm{~min}$ ). After injection, the ganglion was dissected out, fixed in $20 \%$ formaldehyde (pH 3.8 in acetic acid/potassium acetate buffer), dehydrated in alcohol series, cleared in methyl salicylate and mounted on a fluorescence microscope (Olympus, BX-50) for photography. In some preparations, the neuronal structure was optically scanned by a confocal laser scanning microscope (BioRad, Radiance 2100) for more detailed morphological analyses. AF488 could reveal fine processes more reliably than Lucifer Yellow under the laser microscope. 


\section{Results}

We made intracellular recordings from 50 local interneurons responded to statocyst stimulation in the brain. These interneurons were classified into 4 groups (Type-I to IV) depending on the branching pattern of dendrites in the protocerebrum and the deutocerebrum (Table 1). Type-I interneurons extended dendritic branches in the deutocerebrum only on the side ipsilateral to the cell body and in the protocerebrum. Type-II interneurons extended dendrites bilaterally in the deutocerebrum whereas Type-III in the deuto- and protocerebrum. Type-IV interneurons extended dendritic processes bilaterally in the protocerebrum, including non-spiking giant interneurons (NGIs) known as pre-motor interneurons that control eye stalk movements (Yamaguchi and Okada, 1990). The nomenclature of neuropile and cell cluster is based on Tautz and Tautz (1983).

Type-I interneurons

Type-I interneurons had their somata in the ventral paired lateral cluster (Fig. 1a, $b ; n=8$ ) or in the ventral unpaired posterior cluster (Fig. 1c, d; $n=12$ ). They projected dendrites unilaterally (Fig. 1a, c) or bilaterally (Fig. 1b, d) in the protocerebrum.

Interneurons with somata in the ventral paired lateral cluster. They were 
spontaneously active in 7 out of 8 animals. Statocyst stimulation which mimicked body tilting around the longitudinal axis by $90^{\circ}$ (Hama and Takahata 2003) induced a sustained excitatory response in an interneuron that projected dendrites unilaterally on the lowered side (left panel of Fig. 2a). By contrast, the activity of another interneuron with unilateral dendrites on the lifted side was tonically suppressed by the same stimulation. During the excitatory response, the input resistance decreased to about $67.3 \%$ of the resting value while during the suppressive response it increased slightly (right panel of Fig. 2a). These results suggest that these interneurons $(n=4)$ receive sustained depolarizing input by direct synaptic excitation and sustained hyperpolarizing input by synaptic disfacilitation depending on the side of stimulation.

Synaptic responses of those interneurons projecting dendrites bilaterally $(\mathrm{n}=$ 4) are illustrated in Fig. 2b and c. When the animal was at rest, statocyst stimulation induced a phaso-tonic suppressive response (left panel of Fig. 2b). This suppression was found in another interneuron to be caused by a decrease in excitatory input, i.e., synaptic disfacilitation (Fig. 2c). Neither the spontaneous activity nor the synaptic response to statocyst stimulation was affected during active leg movements on a substratum (right panel of Fig. 2b).

The output effect of those interneurons with bilateral dendrites was examined by intracellular injection of depolarizing and hyperpolarizing current into their dendritic processes (Fig. 2d). Electromyographic (EMG) recording was made from the eye-up 
muscle (\#12 in Mellon 1977) on the lifted side. Injection of a depolarizing current into an interneuron increased its spike firing rate to activate the eye up muscle. Hyperpolarizing current injection completely ceased the spike activity of the interneuron, but no noticeable change was induced in the eye-up muscle activity. These physiological characteristics indicate that the interneurons can transmit statocyst signals to the eyestalk motor system independently on leg and behavioral condition.

Interneurons with somata in the ventral unpaired posterior cluster. They showed no spontaneous spike discharge in 9 out of 12 preparations. In response to mimicked body rolling, an interneuron that projected dendrites unilaterally on the lowered side showed transient spike discharges when the animal was at rest (left panel of Fig. 3a). During active leg movements without a substratum, the spontaneous activity and synaptic responses to statocyst stimulation were the same as those observed at rest (middle panel of Fig. 3a). During active leg movements on a substratum, however, the spontaneous activity increased significantly (right panel of Fig. 3a). In this situation, statocyst stimulation completely suppressed the spike firing of the interneuron. No noticeable synaptic response underlying spikes was observed regardless of the behavioral condition of the animal.

By contrast, the mimicked body rolling induced a sustained depolarization superimposed by a few spikes in another interneuron extending dendrites unilaterally on 
the lowered side (left panel of Fig. 3b). When the animal actively moved walking legs on a substratum, a sustained depolarization similar to that observed at rest was induced by statocyst stimulation (middle panel of Fig. 3b). The number of spikes elicited by body rolling, however, was greater in this condition than at rest when the spontaneous spike firing was not affected at all. Active leg movements on a substratum thus selectively affected the synaptic responses to statocyst stimulation. After the statolith was removed unilaterally on the side of stimulation, i.e., the lowered side, the spontaneous activity of the interneuron showed a significant increase when the animal was at rest. The mimicked body rolling that activated statocyst receptors on the intact lifted side caused suppression of this firing (right panel of Fig. 3b). Thus, when the animal body is rolled so that the side of the interneuron is lowered, it receives excitatory and inhibitory input from the statocyst on the lowered and lifted side respectively. In the intact animal, the excitatory input can effectively cancel or override the inhibitory input to elicit spikes superimposed on a sustained depolarization (middle panel of Fig. 3b).

Physiological characteristics of those interneurons extending dendrites bilaterally are illustrated in Fig. 4. One interneuron had its soma on the side contralateral to the magnetic field stimulation, extending dendrites on both sides of the protocerebrum. When the animal was at rest, the interneuron showed no spontaneous spike activity (left panel of Fig. 4a). In response to statocyst stimulation from the opposite side to its soma, a few transient spike discharges were elicited, followed by a 
slight depolarization at the stimulus offset. These characteristics were not affected by leg movements in the air without a substratum (middle panel of Fig. 4a). When the animal actively moved walking legs on the substratum, the interneuron showed a sustained depolarization on which a train of high-frequency spike discharges was superimposed (right panel of Fig. 4a). In this condition, statocyst stimulation completely suppressed this spike firing.

On the other hand, another interneuron extending dendrites bilaterally responded quite differently to statocyst stimulation from the same side with the soma (Fig. 4b), showing a sustained depolarization superimposed by a train of high-frequency spike discharges (left panel of Fig. 4b). When the animal was active and moved walking legs in the air, both the spontaneous activity and the synaptic response to statocyst stimulation were not affected at all (middle panel of Fig. 4b). During active leg movements on a substratum, however, the spike response disappeared almost completely with only the sustained depolarization remaining (right panel of Fig. 4b). Comparison of input resistance changes in response to statocyst stimulation between at rest and during active leg movements on a substratum revealed a complex nature of the synaptic event occurring during this suppression. When the animal was at rest, statocyst stimulation caused a transient decrease in input resistance followed by its gradual increase during the stimulation (upper trace in Fig. 4c). The synaptic input from bilateral statocysts to the interneuron during soma-side-down rolling thus consists of 
early synaptic excitation and later component that tonically depolarizes the cell. Since the input resistance near the end of stimulation appears to be larger than that observed before and after the stimulation, a possible mechanism for the later component would be synaptic disfacilitation as seen in the synaptic input to the interneuron shown above (Fig. 2a). When the animal actively moved walking legs on a substratum (second trace from top in Fig. 4c), the input resistance continuously decreased to about a half of the value observed when the animal was at rest $(\mathrm{P}<0.01$, two-sided Mann-Whitney U-test; Fig. 4d). In this condition, however, neither a transient decrease nor a gradual increase was observed in input resistance.

When the statolith on the lowered side was removed, another interneuron extending dendrites bilaterally showed a similar response to that observed in the intact condition (Fig. 4e). The membrane conductance showed an increase during the synaptic response to mimicked rolling in the intact animal (upper trace pair in Fig. 4e). After the statolith removal, the interneuron also showed an increase in membrane conductance during the synaptic response to body rolling (lower trace pair in Fig. 4e), indicating that the interneuron receives excitatory, not disinhibitory, synaptic input from the statocyst on the lifted side. A slight increase in the response magnitude after the statolith removal suggested that the interneuron receives suppressive input from the statocyst on the lowered side in the intact condition, just opposite to the case of the interneuron shown in Fig. 3b. 
Type-II interneurons

Synaptic responses and structure of a Type-II interneuron $(\mathrm{n}=2)$ are shown in Fig. 5. This interneuron spontaneously showed large depolarizing synaptic activities in the absence of external stimuli when the animal was at rest. Statocyst stimulation that mimicked the soma-side-down rolling induced a small depolarization of about $2 \mathrm{mV}$ in the peak amplitude (left panel of Fig. 5a). When the animal actively moved legs on a substratum, the synaptic response to statocyst stimulation was significantly reduced as well as the amplitude of spontaneous synaptic potentials (middle panel of Fig. 5a). Depolarizing current injection resulted in a decrease of the response to statocyst stimulation whereas hyperpolarization caused its increase (right panel of Fig. 5a), suggesting that the synaptic transmission is mediated chemically.

Intracellular recording from this interneuron was made from the middle part of the thick process connecting the neuritic branches on both sides (Fig. 5b). It characteristically showed large spontaneous synaptic potentials. The small amplitude and slow time course of the synaptic response of this neuron to statocyst stimulation (Fig. 5a) suggested that its synaptic site for statocyst input is electrotonically distant from the recording site, although we could not pinpoint the synaptic location. It was also noted that the time course of individual synaptic potential occurring spontaneously tended to be shorter during active leg movements than during at rest (Fig. 5c), although 
the two populations were not significantly different $(\mathrm{P}>0.05$, two-sided Mann-Whitney U-test). The finding suggested that the synaptic site for spontaneous activities is electrotonically close to that for the leg activity-related input.

\section{Type-III interneurons}

Physiological and structural characteristics of a Type-III interneuron $(n=4)$ are shown in Fig. 6. Two different structural subtypes could be identified for the Type-III interneurons, but their physiological characteristics were the same. Statocyst stimulation mimicking the soma-side-down rolling induced a phasic depolarization without spike firing (left panel of Fig. 6a). This response was unchanged when the animal actively moved legs on a substratum (right panel of Fig. 6a). The interneuron could affect the spike activity of descending interneurons on the lowered side without generating a spike itself (Fig. 6b). Positive current injection (5 nA) resulted in depolarization of the interneuron and a concurrent increase in the spike activity of descending interneurons (upper panel of Fig. 6b). In this situation, no spike firing was observed in this Type-III interneuron. On the other hand, no visible response was induced by negative current (5 nA) injection (bottom panel of Fig. 6b). Magnetic field stimulation caused an increase in the spike activity of descending interneurons on the lowered side in the same preparation (Fig. 6c). It was thus suggested that the interneuron is in series with the descending statocyst interneurons to activate them either directly or polysynaptically. 
Type-IV interneurons

We identified 5 interneurons as Type-IV. Three of them were spiking and others were non-spiking neurons. Since the synaptic responses of spiking interneurons were so diverse, we could not establish their physiological characteristics in this study. Here we report the morphological and physiological characteristics of two non-spiking interneurons. One of them was the non-spiking giant interneuron (NGI) that had been identified by Okada and Yamaguchi (1988). Body rolling elicited a depolarization in the NGIs on the lowered side and a hyperpolarization on the lifted side (Okada et al. 1994). We made intracellular recording from an NGI on the side of stimulation, i.e., on the lowered side during mimicked rolling, and found that it responded to statocyst stimulation with a sustained depolarization (left panel of Fig. 7a). When the animal actively moved legs in the air, the synaptic response of the NGI to statocyst stimulation was remarkably enhanced (middle panel of Fig. 7a). This enhancement, however, was less remarkable during active leg movements on a substratum (right panel of Fig. 7a). The cell structure (Fig. 7c) indicated that the interneuron is either of G1, G2 and G3 that have been reported to be indistinguishable between them and different from G4 and G5 (Okada and Yamaguchi, 1988).

Another type of non-spiking Type-IV interneuron was newly found in this study. Statocyst stimulation from the side opposite to its soma induced a tonic 
depolarization (left panel of Fig. 7b). When the animal actively moved walking legs on a substratum, the synaptic response of the cell was slightly reduced (middle panel of Fig. 7b). The spontaneous synaptic activity was compared between at rest and during active leg movements on the substratum (right panel of Fig. 7b). Hyperpolarizing input was remarkably increased during leg movements, but depolarizing input remained unaffected. Suppression of the synaptic response to statocyst stimulation (middle panel of Fig. 7b) could be partly contributed by this enhancement of the background inhibitory input during leg movements on the substratum. Morphology of this type of cell is shown in Fig. 7d. 


\section{Discussion}

In this study, we classified the local interneurons receiving statocyst input into 4 morphological groups (Type-I to IV) according to their dendritic morphology. Since we found many interneurons so far unknown, we present a new classification to include all statocyst-driven local interneurons in the brain. Type-I interneurons presented here are identical with those previously described by Nakagawa and Hisada (1990). They classified Type-I interneurons to 3 subgroups (ac, vplc and vupc) depending on the soma location. We did not encounter, however, those interneurons that corresponded to the ac subgroup in this study. Our results therefore do not provide a complete catalog of all neuronal components involved in the central statocyst pathway. Nevertheless, the present finding shows an important aspect of the neuronal organization regarding the central statocyst pathway: neurons transmitting statocyst information independently of the leg condition are paralleled by those affected by it (Fig. 8). In the following sections, we discuss the neuronal organization of the central statocyst pathway and its functional significance in the control of body posture and the central compensation process following unilateral statolith removal.

Central pathways of statocyst information

Yoshino et al. (1983) demonstrated by backfill experiments that statocyst sensory afferents project to the parolfactory lobe in the deutocerebrum on the same side, 
some afferents crossing the midline to the same lobe on the opposite side. This lobe corresponds to the lateral antenna I neuropil designated by Sandeman et al. (1992). Type-I and II interneurons had their dendritic projection to this region (Fig. 1 and Fig. 5d), apparently receiving direct input from statocyst afferents on the same side. Some Type-I interneurons also received input from the statocyst on the opposite side (right panel of Fig. 4e). Although the dendritic arborization of Type-I interneurons never crosses the midline, it remains open whether this contralateral statocyst input is mediated monosynaptically by statocyst afferents crossing the midline or polysynaptically via other interneurons.

All Type-I interneurons projected their dendrites to the protocerebrum where many descending interneurons transmitting statocyst information to posterior ganglia extend dendrites (Glantz et al. 1981; Nakagawa and Hisada 1989; Hama and Takahata 2003). The NGIs, which control compensatory movements of eyestalks in response to body tilting, also exist in the protocerebrum with no dendritic projection to the parolfactory lobe (Okada and Yamaguchi 1987). These morphological data suggest that NGIs and descending statocyst interneurons make functional connection with Type-I interneurons in the protocerebrum to receive statocyst sensory information. Type-III interneurons, extending dendrites in the parolfactory lobe of the deutocerebrum and the lateral optic lobe of the protocerebrum, are also candidate mediators of statocyst information to NGIs and descending interneurons (Fig. 8). 
It is interesting to note here that the compensatory eye movement system, potentially the simplest sensori-motor pathway from statocyst receptors to eye motor neurons on the same side, is organized polysynaptically with at least 2 intervening neurons including NGIs. The result of cobalt backfilling experiments that many of the eyecup motor neurons extend dendrites to the protocerebrum as well as to the deutocerebrum (Mellon 1977) supports this possibility. Even at the first stage of the sensori-motor pathway, the synaptic input to Type-I interneurons is not simple. Comparison of the synaptic responses of a Type-I interneuron to body rolling before and after statolith removal on the lowered side (Fig. 3b) indicates that during body rolling the statocyst on the lowered side supplies excitatory signals whereas that on the lifted side inhibitory ones. This observation is consistent with the previous report on the synaptic input of NGIs (Okada et al. 1994). However, we also found that excitatory synaptic responses accompanied by an increase in membrane conductance were induced in another Type-I interneuron receiving input from the statocyst on the lifted side during body rolling in an animal with its statolith removed on the lowered side (Fig. 4e). This finding indicates that the statocyst on the lifted side can supply excitatory or inhibitory signals to Type-I interneurons depending on the cell.

In this study, we could not examine whether the depolarizing response evoked by the statocyst on the lowered side (Fig. 3b) was accompanied by an increase or a decrease in membrane conductance. It remains open, therefore, whether the response is 
due to synaptic excitation or disinhibition. As shown in Fig. 2a and c, Type-I interneurons themselves can be of second-order to the statocyst afferents. In sum, the polysynaptic organization of the statocyst pathway indicates that it can be critically affected by other sensory input as well as motor command-related central input (Furudate et al. 1996; Fraser and Takahata 2002) as in other crustacean sensori-motor systems in general (Sandeman 1978; Hamm and Tautz 1990). Although the central input to the statocyst pathway is not incorporated in the hypothetical wiring diagram (Fig. 8), the behavioral context dependence of the pathway function is represented by converging input of statocyst and proprioceptor information to Type-I, -II and -IV interneurons that is in contrast with selective input of statocyst information to Type-I and Type-III interneurons

Parallel organization of the statocyst central pathway

Our previous report proposed that many statocyst-driven descending interneurons (SDIs) receive neural signals related to execution of leg movements so that they are activated by body rolling differently between at rest and during free leg movements (Hama and Takahata 2003). In this study, we showed that the synaptic responses of Type-I and III interneurons to statocyst stimulation are not affected when the animal actively moves legs in the air without a substratum (Fig. 3, 4 and 5). The responses of Type-IV interneurons, by contrast, were clearly affected by free leg 
movements (Fig. 5). As discussed in our previous study (Hama and Takahata, 2003), the difference between active leg movements on a substratum and in the air appears in the brain as a difference in the balance between sensory signals from leg proprioceptors (Cattaert et al. 1990, 1992; El Manira et al. 1991) and central signals from the locomotor center (von Holst and Mittelstaedt 1950; Sperry 1950). It is interesting to note here that Type-IV interneurons that were affected by leg movements in the air were structurally confined to within the protocerebrum whereas other types of interneurons that were not affected by free leg movements projected dendritic processes in both the protocerebrum and the deutocerebrum. It remains unknown, however, where in the brain the leg proprioceptor pathway makes interaction with the statocyst system as well as the central pathway carrying the corollary discharge signals. Although physiological evidence indicates that leg proprioceptor signals ascend to the brain (Wiersma 1958), no information is available on their projection site.

It should be noted here that some of the Type-I interneurons, candidate mediators of statocyst information to NGIs, show invariant responses to statocyst stimulation regardless of the leg condition of the animal (Fig. 2). But many other Type-I interneurons (Figs. 3, 4) that transmit statocyst information from the deutocerebrum to the protocerebrum were facilitated during leg movements on a substratum except the one shown in Fig. 4d that showed response depression in that leg condition. These results suggest that NGIs receive statocyst information by way of 
leg-condition-independent pathways through Type-I interneurons shown in Fig.2 and Type-III interneurons (Fig. 6), and leg-condition-dependent pathways through other Type-I interneurons (Figs. 3, 4). Judging from their location in the protocerebrum, the Type-IV interneuron whose synaptic response to statocyst stimulation is affected by the leg condition (Fig. 7b) are also involved in the synaptic pathway from statocyst afferents to NGIs. The variable response of NGIs to statocyst stimulation is thus due, at least partly, to variable responses of pre-NGI neurons that are dependent on the leg condition of the animal. The variability in the synaptic response of NGIs to statocyst stimulation strongly suggests that the compensatory eyestalk movements will be affected, as other equilibrium responses, by the animal's behavioral condition.

The functional role of Type-II interneurons in the central statocyst pathway is unknown. It has been shown that the central compensation, in which the bilaterally asymmetrical posture of appendages following unilateral statolith removal is gradually normalized to the original symmetrical configuration in a few weeks (Schöne 1954; Yoshino et al 1980), occurs more successfully when the experimental animal is provided with a substratum than not during the post-operative period (Sakuraba and Takahata 1999, 2000). Free leg movement without a substratum was found to be insufficient for successful attainment of central compensation. These results indicate that the central compensation process crucially depends on the leg condition after operation. Our finding that the synaptic responses of statocyst-driven local interneurons except some of 
the Type-I interneurons (Fig. 2) and Type-III interneurons (Fig. 6) were critically affected by leg conditions suggests their potential function in the central compensation process. Although we could not determine which type of local interneurons are central to the compensation, we propose that Type-II interneurons, connecting the statocyst afferent terminal on both sides, should be examined with great care because the inhibitory connection connecting the vestibular nucleus on both sides (Shimazu and Precht 1966) plays an important role in the vestibular compensation after unilateral labyrinthectomy of vertebrates (Dieringer 1995; Smith and Curthoys 1989; Graham and Duita 2001). The postsynaptic action of Type-II interneurons is unknown. But their role as a relay pathway over the midline appears to be unlikely since statocyst sensory afferents partly project into the parolfactory lobe of deutocerebrem on the contralateral side (Yoshino et. al. 1983). Our observation that an interneuron extending dendrites only on the soma side receives inhibitory input from contralateral statocyst (Fig. 3b) suggests that the input is mediated by Type-II interneurons.

The dendritic arborization of Type-II interneurons, confined to within the deutocerebrum on both sides, suggests their functional role as the distributor of statocyst sensory signals over the midline at the early stage of sensory information processing. Having bilateral dendrites, Type-III and IV interneurons are therefore also candidate distributor of statocyst information over the midline, but Type-II interneurons are, specialized as such a distributor, situated at the most sensory level, i.e., at the first- or 
second-order level. By contrast, Type-IV interneurons including NGIs that directly control the activity of eyestalk motoneurons (Yamaguchi and Okada 1990) seem to be situated at or near the premotor level in the sensori-motor pathway. It is thus suggested that the statocyst signal traffic over the midline at various levels along the sensori-motor pathway subserves the bilaterally coordinated equilibrium reflexes (Schöne 1951; Davis 1968; Schöne et al. 1976; Yoshino et al. 1980). 


\section{Acknowledgements}

This work was partially supported by grants (Nos. 14340260, 15657018) from the Ministry of Education, Science, Culture and Sports, Japan. 


\section{References}

Alverdes F (1926) Stato-, Photo- und Tangoreaktionen bei zwei Garneelenarten. Z vergl

Physiol 4: 699-765

Cattaert D, El Manira A, Clarac F (1992) Direct evidence for presynaptic inhibitory mechanisms in crayfish sensory afferents. J Neurophysiol 67: 610-624

Cattaert D, El Manira A, Marchand A, Clarac F (1990) Central control of the sensory afferent terminals from a leg chordotonal organ in crayfish in vitro preparation. Neurosci Lett 108:81-87

Davis WJ (1968) Lobster righting responses and their neuronal control. Proc R Soc Lond B 170: 435 - 456

Davis WJ, Mpitsos GJ, Pinneo JM (1974) The behavioral hierarchy of the mollusk Pleurobranchaea. I. The dominant position of the feeding behavior. J Comp Physiol 90: 207-224

Deliagina TG, Orlovsky GN, Selverston AI, Arshavsky YI (1999) Neuronal mechanisms for the control of body orientation in Clione I. Spatial zones of activity of different neuron groups. J Neurophysiol 82: 687- 699

Deliagina TG, Zelenin P, Fagerstedt P, Grillner S, Orlovsky GN (2000) Activity of reticulospinal neurons during locomotion in the freely behaving lamprey. $\mathrm{J}$ Neurophysiol 83: 853-863

Dieringer N (1995) “Vestibular compensation.” Neuronal plasticity and its relations to 
functional recovery after labyrinthine lesion in frogs and other vertebrates. Prog Neurobiol 46: 97-129

Everett RA, Ostfeld RS, Davis WJ (1982) The behavioral hierarchy of the garden snail Helix aspersa. Z Tierpsychol 59: 109-126

Fraser PJ, Bevengut M, Clarac F (1987) Swimming patterns and the activity of identified equilibrium interneurons in the shore crab, Carcinus maenas. J Exp Biol 130: 305-330

Fraser PJ, Takahata M (2002) Statocysts and statocyst control of motor pathways in crayfish and crabs. In: Crustacean Experimental Systems in Neurobiology (ed Wiese K), Springer, Berlin pp89-108

Furudate H, Okada Y, Yamaguchi T (1996) Responses of nonspiking giant interneurons to substrate tilt in the crayfish, with special reference to multisensory control in the compensatory eyestalk movement system. J Comp Physiol A 179: 635-643

Glantz RM, Kirk M, Viancour T (1981) Interneurons of the crayfish brain: the relationship between dendrite location and afferent input. J Neurobiol 12: 311-328.

Graham BP, Duita MB. (2001) Cellular basis of vestibular compensation: analysis and modeling of the role of the commissural inhibitory system. Exp Brain Res 137: 387-396 
Hama N, Takahata M (2003) Effects of leg movements on the synaptic activity of descending statocyst interneurons in crayfish, Procambarus clarkii. J Comp Physiol A 189: 877-888.

Hamm I, Tautz J (1990) Locomotion modulates the sensitivity of mechanosensory interneurons in freshwater crayfish. In: Frontiers in Crustacean Neurobiology (ed Wiese K, Krenz W -D, Tautz J, Reichert H, Mulloney B) Springer, Berlin pp152-156

Harreveld A van (1936) A physiological solution for freshwater crustaceans. Proc Soc Exp Biol Med 34: 428-432

Holst E von, Mittelstaedt H (1950) Das Reafferenzprinzip: Wechselwirkungen zwischen Zentralnervensystem und Peripherie. Naturwissenschaften 37: 464 - 476

Horak FB, Macpherson JM (1996) Postural orientation and equilibrium. In: Handbook of Physiology. Exercise: Regulation and Integration of Multiple Systems. Oxford University Press, New York sect 12 pp 255-292

Kühn A (1914) Die reflektorische Erhaltung des Gleichgewichtes bei Krebsen. Verh Dt Zool Ges 24: 262-277

Mellon DeForest Jr. (1977) The anatomy and motor nerve distribution of the eye muscle in the crayfish. J. Comp. Physiol. A 121: 349-366

Nakagawa H, Hisada M (1989) Morphology of descending statocyst interneurons in the crayfish Procambarus clarkii Girard. Cell Tissue Res 255: 539-551 
Nakagawa H, Hisada M (1990) Local spiking interneurons controlling the equilibrium response in the crayfish Procambarus clarkii. J Comp Physiol A 170: 291-302

Okada Y, Yamaguchi T (1988) Nonspiking giant interneurons in the crayfish brain: morphological and physiological characteristics of the neurons postsynaptic to visual interneurons. J Comp Physiol A 162: 705-714.

Okada Y, Furudate H, Yamaguchi T (1994) Multimodal responses of the nonspiking giant interneurons in the brain of the crayfish Procambarus clarkii. J Comp Physiol A 174: 411-419

Ozeki M, Takahata M, Hisada M (1978) Afferent response patterns of the crayfish statocyst with ferrite grain statolith to magnetic field stimulation. J. Comp. Physiol. A123: 1-10

Sakuraba T, Takahata M (1999) Effects of visual and leg proprioceptor inputs on recovery of eyestalk posture following unilateral statolith removal in the crayfish. Naturwissenschaften 86: 346-349

Sakuraba T, Takahata M (2000) Motor pattern changes during central compensation of eyestalk posture after unilateral statolith removal in crayfish. Zool Sci 17: $19-26$

Sandeman DC (1978) Eye-scanning during walking in the crab Leptograpsus variegatus. J. Comp. Physiol. A 124: 249-257

Sandeman D, Sandeman R, Derby C, Schmidt M (1992) Morphology of the brain of 
crayfish, crabs, and spiny lobster: A common nomenclature for homologous structure. Biol. Bull. 183: 304-326

Schöne H (1954) Statocystenfunktion und statische Lageorientierung bei dekapoden Krebsen. Z vergl Physiol 36: 241-260

Schöne H, Neil DM, Stein A, Carlstead MK (1976) Reactions of the spiny lobster, Palinurus vulgaris, to substrate tilt (I.). J Comp Physiol 107: 113-128

Schöne H, Neil DM, Scapini F and Dreismann G (1983) Interaction of substrate, gravity and visual cues in the control of compensatory eye responses in the spiny lobster, Palinurus vulgaris. J Comp Physiol A 150: 23-30

Shimazu H, Precht W (1966) Inhibition of central vestibular neurons from the contralateral labyrinth and its mediating pathway. J Neurophysiol 29: 467-492

Smith PF, Curthoys IS (1986) Mechanisms of recovery following unilateral labyrinthectomy: a review. Brain Res Rev 14: 155-180

Sperry RW (1950) Neuronal basis of the spontaneous optokinetic response produced by visual inversion. J Comp Physiol Psychol 43: 482-489

Takahata M, Hisada M (1982) Statocyst interneurons in the crayfish Procambarus clarkii Girard. I. Identification and response characteristics. J Comp Physiol A 149: $287-300$

Takahata M, Komatsu H, Hisada M (1984) Positional orientation determined by the behavioural context in Procambarus clarkii Girard (Decapoda: Macrura) 
Behaviour 88: 240-265

Takahata M, Murayama M (1992) Multiple gate control of the descending statocyst-motor pathway in the crayfish Procambarus clarkii Girard. J. Comp. Physiol. 170: 463-477

Tautz J, Tautz RM (1983) Antennal neuropile in the brain of the crayfish: Morphology of neurons. J. Comp. Neurol. 218: 415-425

Ullén F, Deliagina TG, Orlovsly GN, Grillner S (1995) Spatial orientation in lamprey. II. Visual influence on orientation during locomotion and in the attached state. $\mathrm{J}$ Exp Biol 198: 675 - 681

Wiersma CAG (1958) On the functional connections of single units in the central nervous system of the crayfish, Procambarus clarkii (Girard). J. Comp. Neurol. 110: $421-471$

Yamaguchi T, Okada Y (1990) Giant brain neurons of the crayfish: Their functional roles in the compensatory oculomotor system. In: Frontiers in Crustacean Neurobiology (ed Wiese K, Krenz W -D, Tautz J, Reichert H, Mulloney B) Springer, Berlin pp193-199

Yoshino M, Kondoh Y, Hisada M (1983) Projection of statocyst sensory neurons associated with crescent hairs in the crayfish Procambarus clarkii Girard. Cell Tissue Res. 230: 37-48

Yoshino M, Takahata M, Hisada M (1980) Statocyst control of uropod movement in 
response to body rolling in crayfish. J Comp Physiol A 139: 243-250 


\begin{tabular}{ccccc} 
& \multicolumn{2}{c}{ Projection } & \multicolumn{2}{c}{ Physiology } \\
\cline { 2 - 5 } Morphological types & deutocerebrum & protocerebrum & $\begin{array}{c}\text { leg movements on a } \\
\text { substratum }\end{array}$ & $\begin{array}{c}\text { leg movements in } \\
\text { the air }\end{array}$ \\
\hline Type-I & unilateral & uni- or bilateral & Y or N* & $\mathrm{N}$ \\
Type-II & bilateral & no projection & $\mathrm{Y}$ & - \\
Type-III & bilateral & unilateral & $\mathrm{N}$ & $\mathrm{N}$ \\
Type-IV & no projection & bilateral & $\mathrm{Y}$ & $\mathrm{Y}$ \\
\hline
\end{tabular}

Table 1 Summary of structural and physiological characteristics of four groups of interneurons identified in this study. * Type-I interneurons having somata in the ventral unpaired posterior cluster were affected by leg movements on a substratum whereas those having somata in the ventral paired lateral cluster were not. The effect of leg movements without a substratum in the air could not be studied for Type-II interneurons. 
a

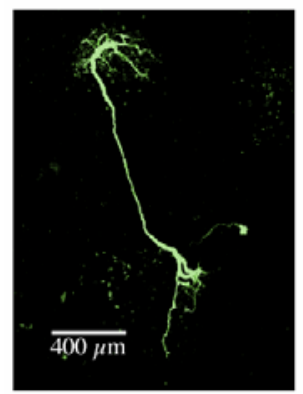

$\mathrm{c}$

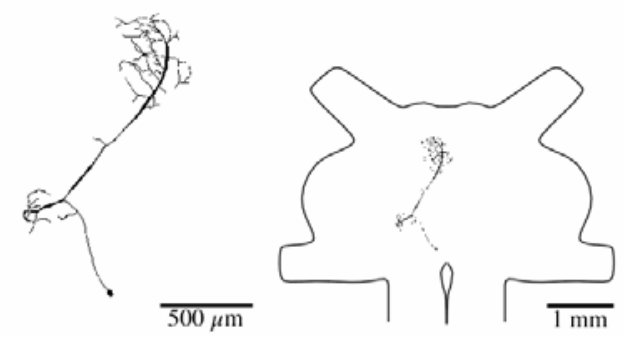

b

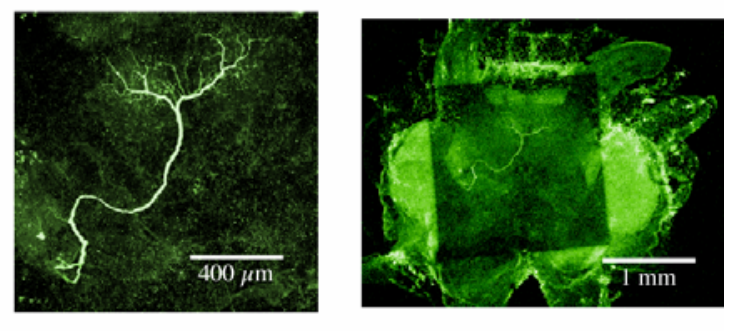

d

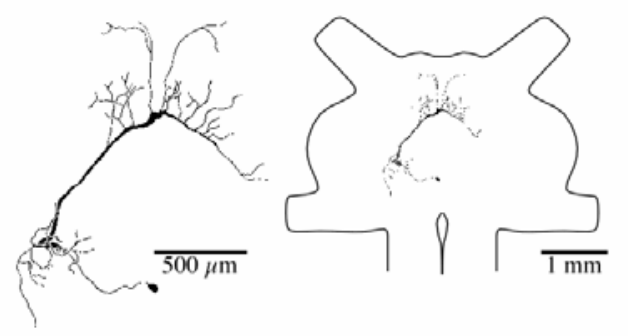

Figure-1

Figure 1 Structure of Type-I interneurons a, b: Interneurons having their somata in the ventral paired lateral cluster. c, d: Interneurons with their somata located in the ventral unpaired posterior cluster. All Type-I interneurons had dendritic projection into the ventral side of the deutocerebrum, i.e., the parolfactory lobe where statocyst afferents project. 
a

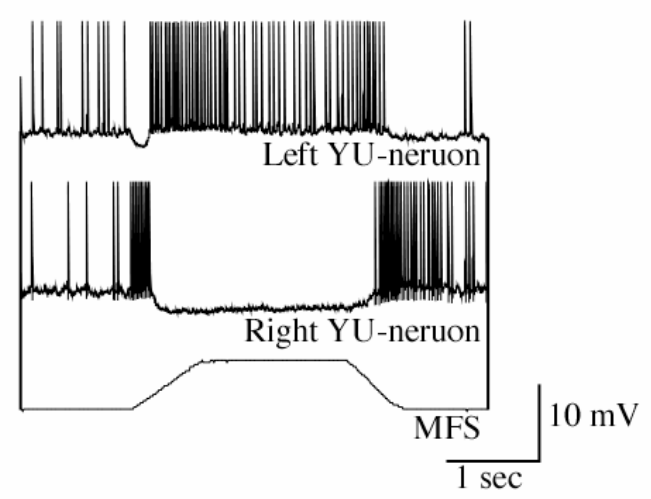

b

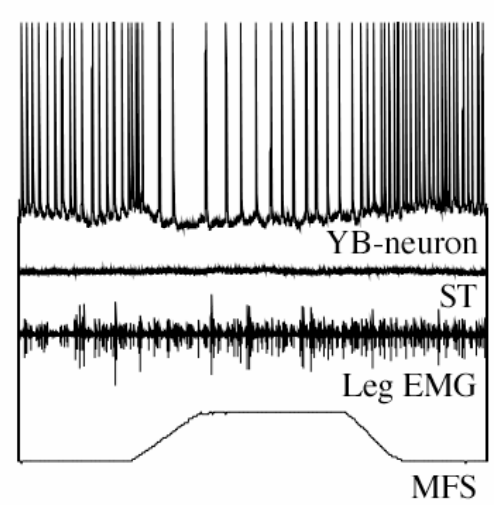

c

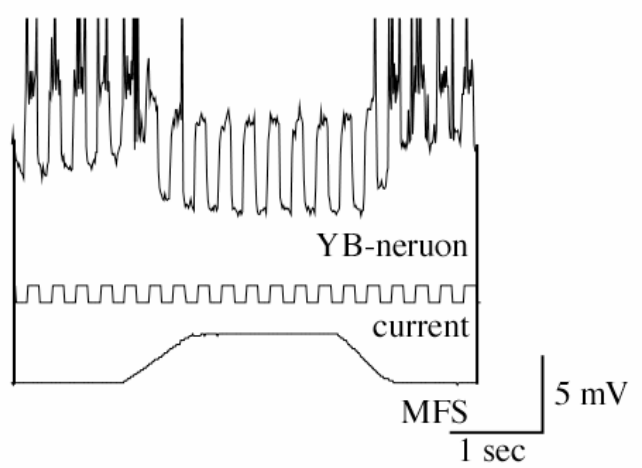

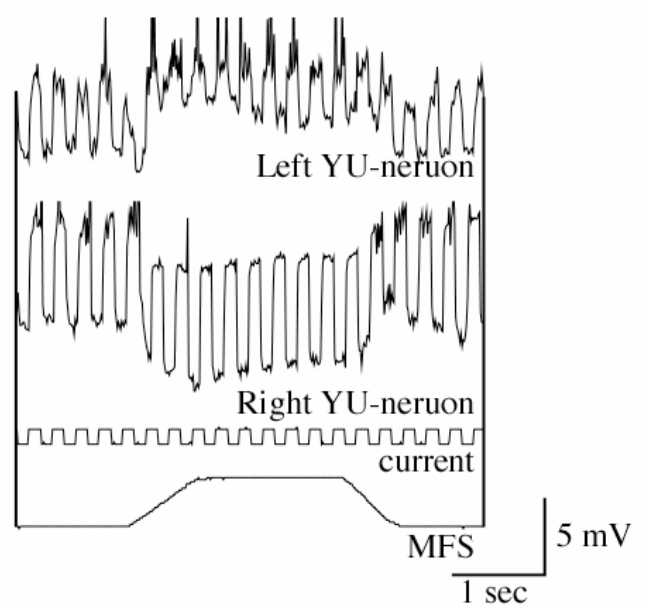
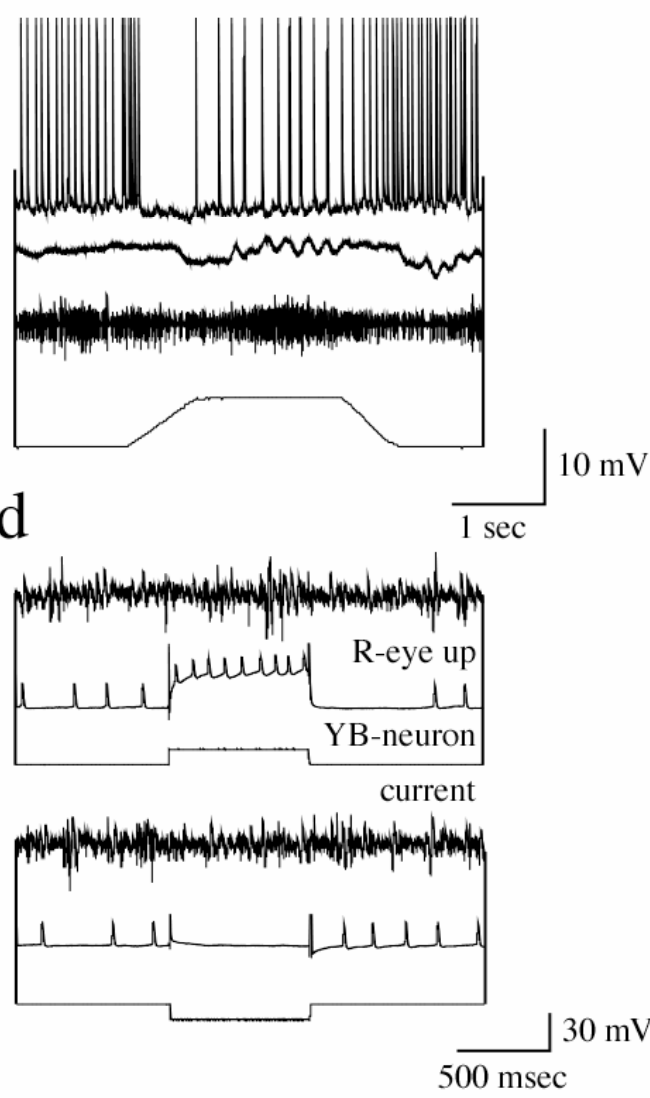

\section{Figure-2}


Figure 2 Physiological characteristics of Type-I interneurons having somata in the ventral paired lateral cluster. a: Interneurons that extend dendrites unilaterally. We could confirm their directional sensitivity in the response to mimicked body rolling. It showed depolarization on the lowered side and hyperpolarization on the lifted side (left panel). Recording was made from different animals for each side. The membrane conductance showed an increase during depolarization and a slight decrease during hyperpolarization in response to statocyst stimulation (right panel). b: Interneurons that extend dendrites bilaterally. Statocyst stimulation decreased the spontaneous spike activity (left panel). The response was not altered during active leg movements on a substratum (right panel). Top trace, intracellular record (IN); second trace, output from the transducer monitoring leg movements on a substratum (ST); third trace, leg EMG; bottom trace, current for magnetic field stimulation (MFS). c: Decrease in membrane conductance during the synaptic response to statocyst stimulation. d: Output connection. Depolarizing current injection into another interneuron resulted in an increase in the eye up muscle activity on the side ipsilateral to its soma. 

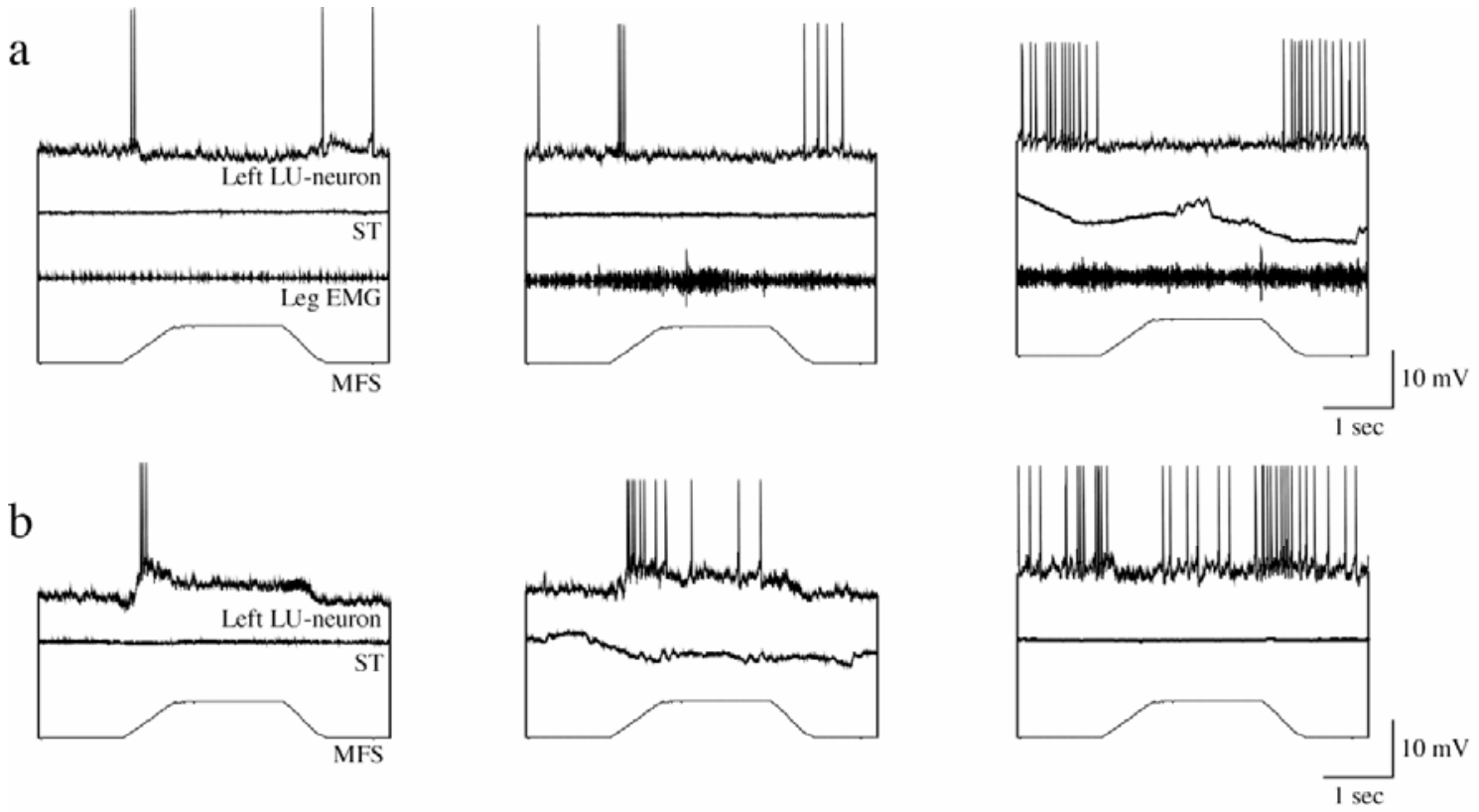

Figure-3 
Figure 3 Effects of leg condition on the statocyst input to Type-I interneurons that have somata in the ventral unpaired posterior cluster and extend dendrites unilaterally. a: An interneuron on the lowered side, receiving transient excitatory input at rest (left panel). When the animal actively moved legs in the air, there was no change in the spontaneous activity and response to statocyst stimulation (middle panel). Active leg movements on a substratum induced a sustained excitation to increase the spontaneous spike activity. Statocyst stimulation suppressed the spike activity instead of transient excitation (right panel). b: Another interneuron on the lowered side, receiving a sustained depolarization with transient spike discharges at rest (left panel). During active leg movements on a substratum, the spike response was enhanced although the sustained depolarizing input was not affected (middle panel). After unilateral statolith removal, the spontaneous spike activity increased while body rolling in the operated-side-down direction resulted in suppression of the spike firing (right panel). 

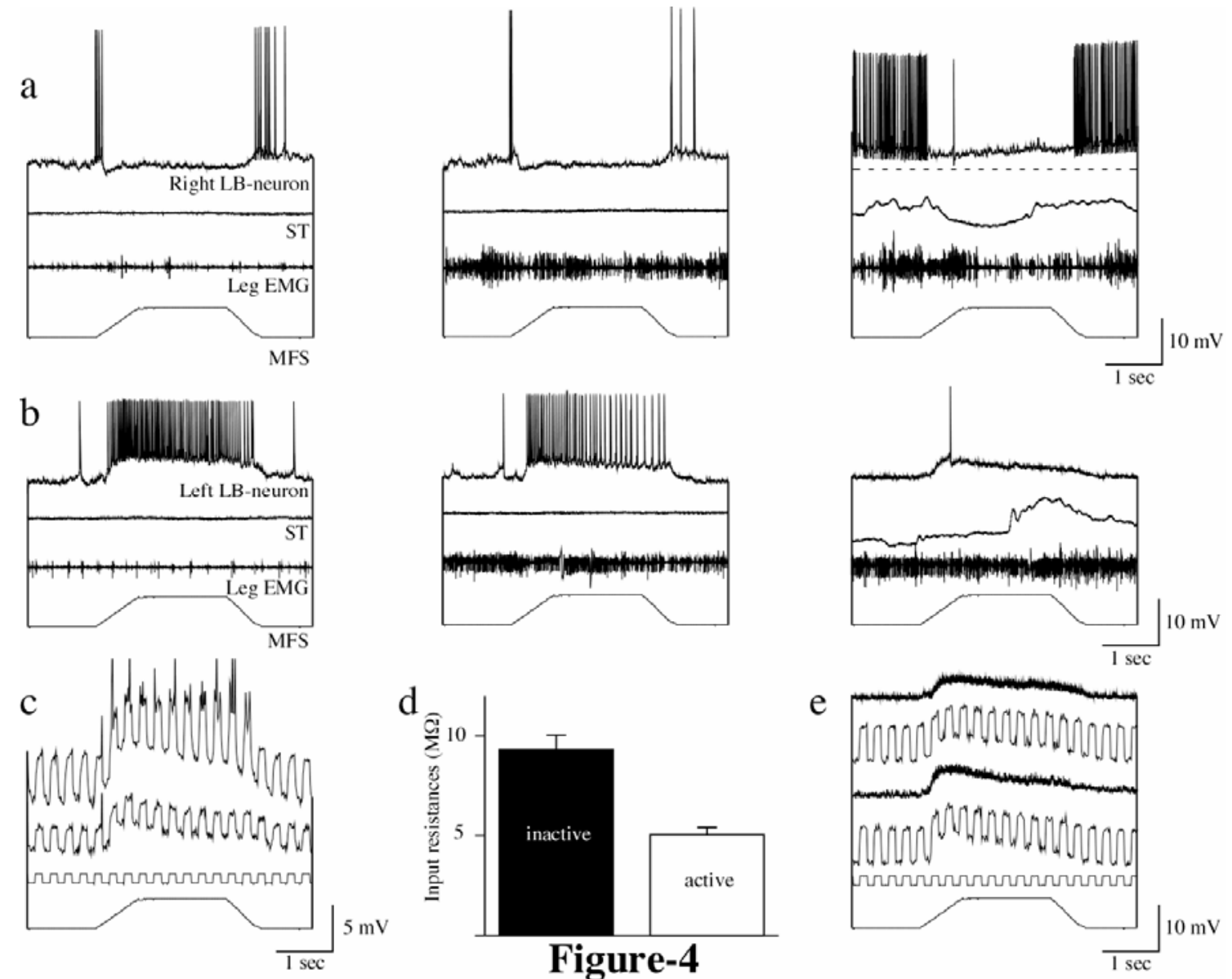
Figure 4 Effects of leg condition on statocyst input to Type-I interneurons that have somata in the ventral unpaired posterior cluster and extend dendrites bilaterally. a: An interneuron on the lifted side, receiving transient excitatory input at rest (left panel). Neither spontaneous spike activities nor synaptic responses were affected by leg movements without a substratum (middle panel). During active leg movements on a substratum, the spontaneous spike activity increased and its suppression was observed in response to statocyst stimulation (right panel). b: Another interneuron on the lowered side, receiving a sustained depolarization superimposed with spike discharges (left panel). When the animal actively moved legs in the air, both spontaneous spike activities and synaptic responses were similar to those observed at rest (middle panel). If a leg substratum was provided, the statocyst stimulation induced a sustained depolarization as before, but the spike discharge was suppressed (right panel). c: Membrane conductance change before and during statocyst stimulation. When the animal was at rest, the membrane conductance showed an initial decrease followed by gradual increase during stimulation (upper trace). During active leg movements on a substratum, the membrane conductance was unchanged before and during stimulation (lower trace). The bottom trace monitors injected current pulses. d: Statistical comparison of input resistance before stimulation between at rest and during active leg movements on a substratum. e: Excitatory input to another interneuron from the contralateral statocyst. Recorded from a different animal. When the statolith was intact 
on both sides, the mimicked body rolling elicited a sustained depolarization (upper trace). After statolith removal on the lowered side, the sustained depolarization remained unchanged and the membrane conductance showed an increase during depolarization (lower trace). 


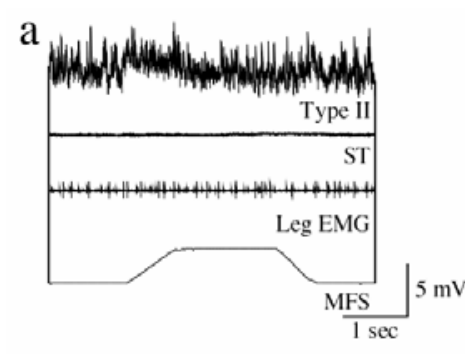

b

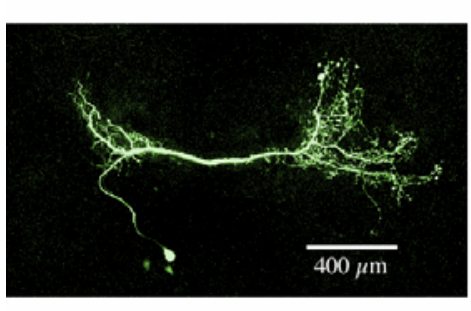

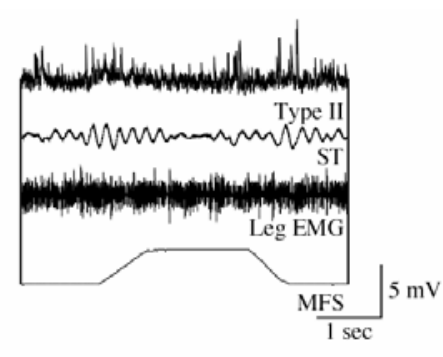

c

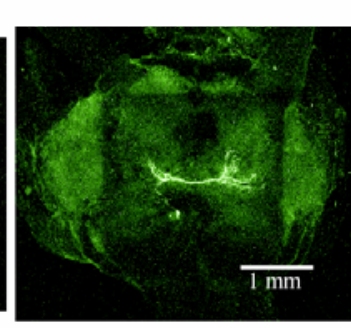

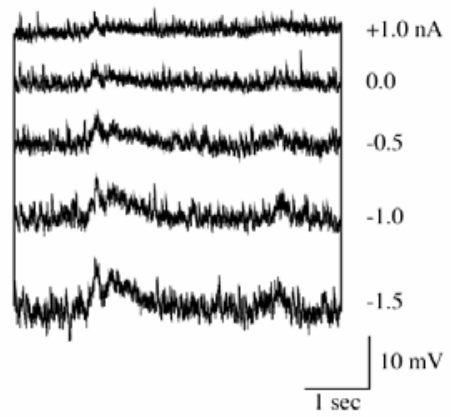

inactive active

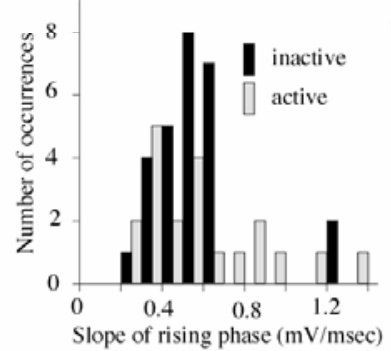

$N \Omega$

$\wedge \Lambda$

$\Omega u$

$\Upsilon_{0.5 \mathrm{msec}} 2 \mathrm{mv}$

\section{Figure-5}

Figure 5 Physiological and morphological characteristics of Type-II interneurons. a: Effects of leg condition on the synaptic responses to statocyst stimulation. A small depolarization was elicited by statocyst stimulation at rest (left panel). This response was reduced when the animal actively moved legs on a substratum (middle panel). The synaptic response could be enhanced by hyperpolarizing current injection and decreased by depolarization (right panel). b: Detailed structure and location in the brain of the interneuron whose responses are shown in a. c: Comparison of synaptic activities between at rest and during active leg movements on a substratum. The slope of rising phase in individual synaptic potential tended to be greater during leg movements than at rest, although the difference was not significant statistically $(\mathrm{P}>0.05$; Mann-Whitney's two-sided U-test). 


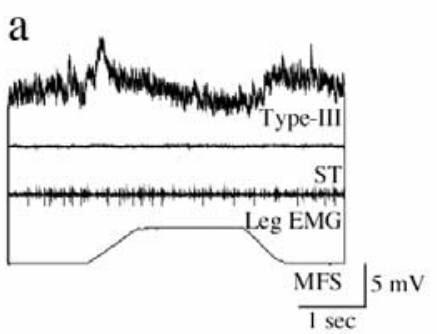

c

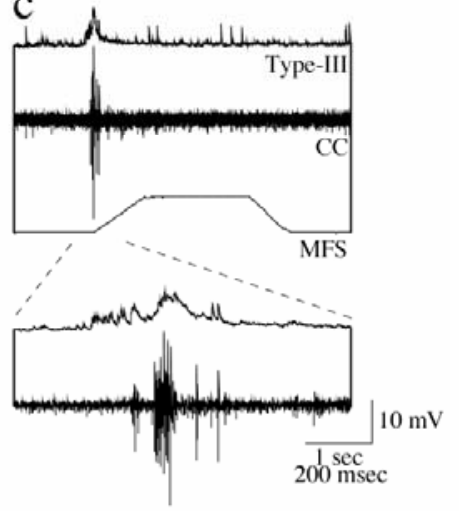

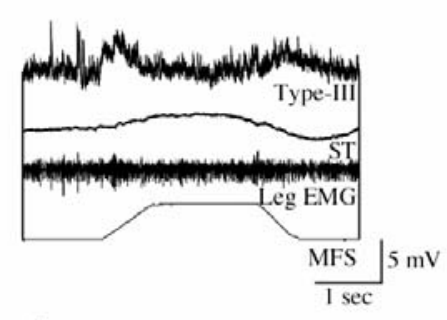

d

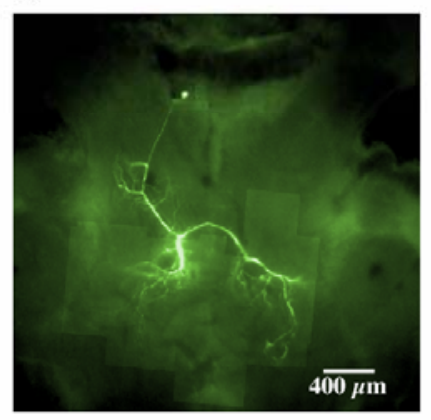

b
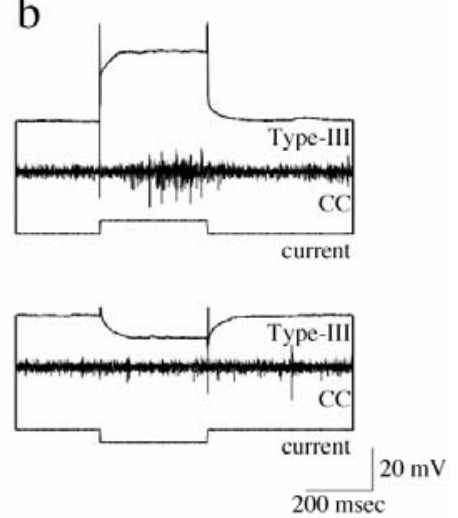

Figure-6 
Figure 6 Physiological and morphological characteristics of Type-III interneurons. a: Effects of leg condition on the synaptic responses of a Type-III interneuron to statocyst stimulation that induced a phasic depolarization at rest (left panel). A similar depolarizing response was observed when the animal actively moved walking legs on a substratum (right panel). b: Output effects of another interneuron. Depolarization by intracellular current injection (5 nA), monitored by the bottom trace, increased the spike activity of descending pathways as monitored extracellularly at the circumesophageal commissure (CC, second trace). Hyperpolarization (-5 nA) caused no effect on the descending activity. c: Simultaneous recording from another Type-III interneuron and descending axons (CC). Statocyst stimulation caused depolarizing synaptic potentials in the interneuron and spike discharges in descending interneurons. Responses are expanded in time scale below. d: Structure of the interneuron shown in a and b. 
a

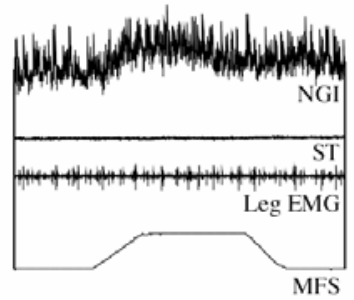

b

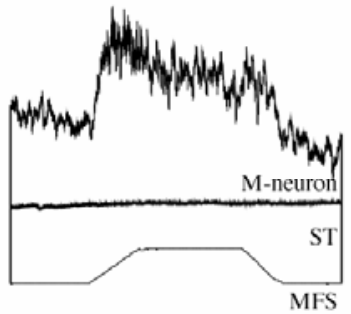

c

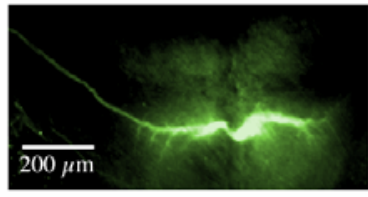

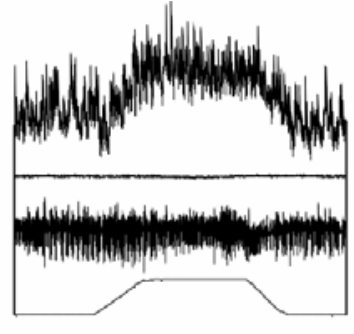
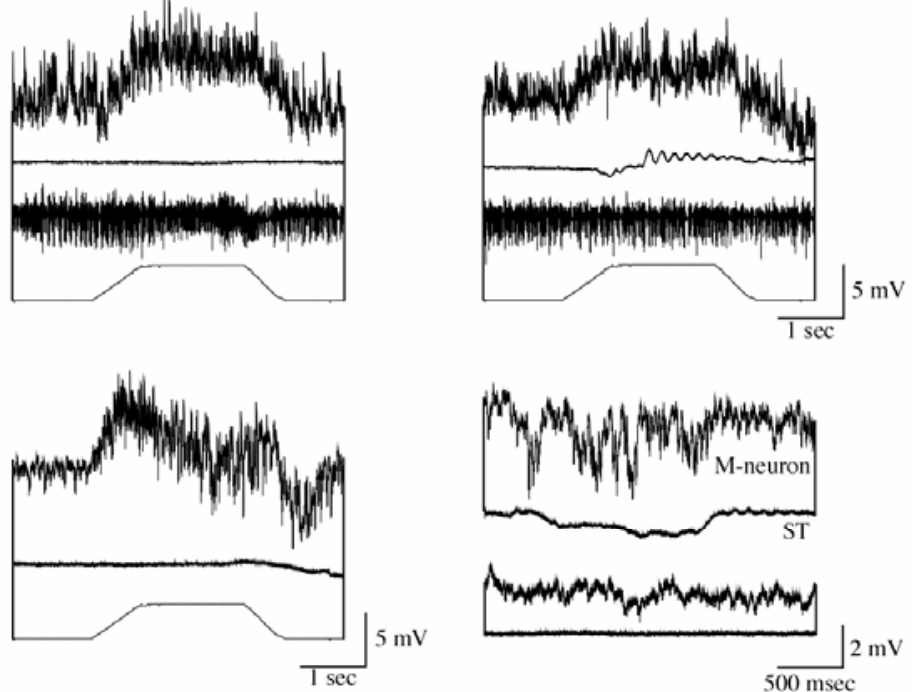

d

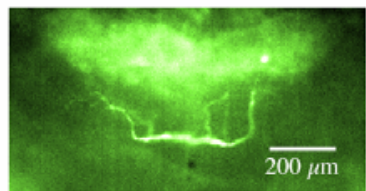

Figure-7 
Figure 7 Physiological and morphological characteristics of Type-IV interneurons. a: Effects of leg condition on synaptic responses of an NGI to statocyst stimulation. A slight depolarization was induced by statocyst stimulation at rest (left panel). When the animal actively moved legs in the air, the responses were significantly enhanced (middle panel). This enhancement was reduced when the animal actively moved legs on a substratum (right panel). b: Effects of leg condition on synaptic responses of another Type-IV interneuron. An excitatory response without spike discharge was elicited when statocyst was stimulated at rest (left panel). During active leg movements on a substratum, the responses to statocyst stimulation were slightly decreased (middle panel). In this situation, the fluctuation of membrane potential due to synaptic activities was larger than that observed at rest. c: Structure of the NGI shown in a. d: Structure of the interneuron shown in b. 


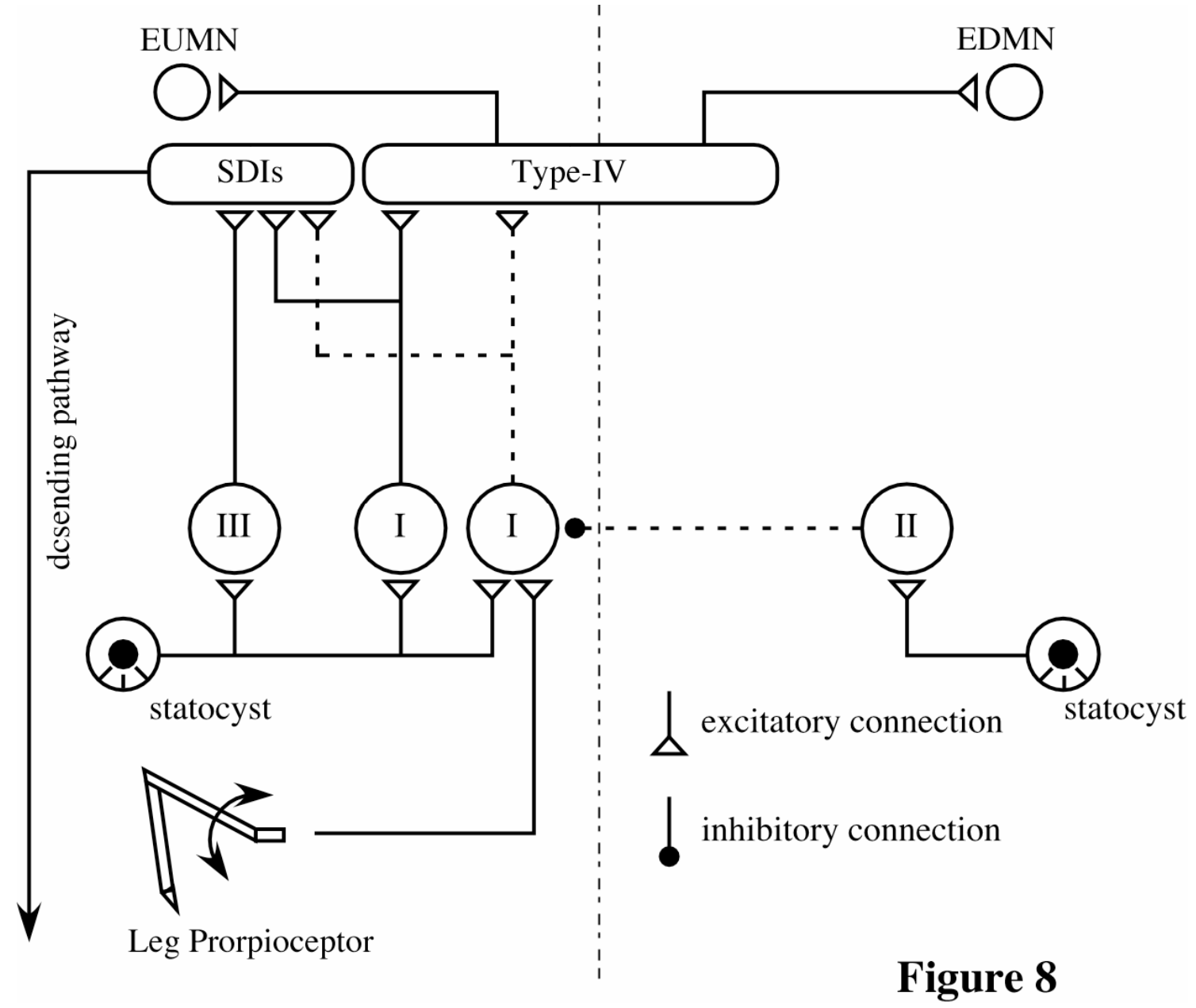


Figure 8 Schematic representation of the central statocyst pathway deduced from the morphological and physiological data presently obtained. Not all connections revealed in this study are shown in this diagram. It shows that the central statocyst pathway is organized in parallel, one that is critically affected by leg condition including some Type-I interneurons (indicated by dashed lines) and the other not affected at all (solid lines) including other Type-I interneurons and Type-III interneurons, leading to motor output through Type-IV interneurons including NGIs (Okada and Yamaguchi, 1988), and statocyst descending interneurons (SDIs, Hama and Takahata, 2003) in the protocerebrum. Type-II interneurons are assumed to function in commissural inhibition between bilateral parolfactory lobes in the deutocerebrum. For the sake of clarity, not all connections are shown in this diagram, including the excitatory connection from the contralateral statocyst to Type-I interneurons as illustrated in Fig. 4e. EDMN, Eyecup-down motor neurons; EUMN, Eyecup-up motor neurons. 